\title{
ACRONYMS AND ORGANIZATIONS
}

AFASDA. Association Femmes Soleil d'Haiti; civil society organization

AVSI. Association of Volunteers in International Service; international NGO

CEDAW. Convention on the Elimination of All Forms of Discrimination against Women

CHREPROF. Centre Haïtien de Recherches et d'Actions pour la Promotion Féminine

DDR programs. disarmament, demobilization, and reintegration programs

ENFOFANM. Organization for the Defense of Women's Rights

GHESKIO. Le Groupe Haïtien d'Etude du Sarcome de Kaposi et des Infections Opportunistes/Haitian Group for the Study of Kaposi's Sarcoma and Opportunistic Infections; national NGO

IBERS. Institut du Bien-Etre Social et de la Recherche/Institute of Social Well-Being and Research

ICRC. International Committee of the Red Cross

IFES. International Foundation for Electoral Systems

IOM. International Organization for Migration

IRB. Institutional Review Board

Kay Fanm. civil society organization

KOFAVIV. Komisyon Fanm Viktim pou Viktim/Commission of Women Victims for Victims; civil society organization 
Kopadim. civil society organization

MDM. Médecins du Monde

MINUSTAH. United Nations Stabilization Mission in Haiti

Mouvement des Femmes Haitiennes. Haitian Women's Movement

MSF. Médecins sans Frontières/Doctors without Borders

NGO. non-governmental organization

OAS. Organization of American States

OZANFAN. Organizasyon Zanmi Fanmi; civil society organization

Padre Stra. religious organization

PNH. Haitian National Police

SOFA. Solidarity Fanm Ayisyen; civil society organization

Suor Anna. religious organization

Tribunal des Mineurs. Tribunal for Minors

UNDP. United Nations Development Programme

UNFPA. United Nations Population Fund

UNICEF. United Nations International Children's Emergency Fund

UNIFEM. United Nations Development Fund for Women

UNPOL. United Nations Police Department

UNSMIH. United Nations Support Mission in Haiti

URAMEL. Unité de Recherche et de Action Médico Légale

Viva Rio. international NGO

WFP. World Food Program

WHO. World Health Organization 
Gender and Violence in Haiti 
